\title{
Tau Protects Microtubules in the Axon from Severing by Katanin
}

\author{
Liang Qiang, ${ }^{1 *}$ Wenqian Yu, ${ }^{1 *}$ Athena Andreadis, ${ }^{2}$ Minhua Luo, ${ }^{2}$ and Peter W. Baas ${ }^{1}$ \\ ${ }^{1}$ Department of Neurobiology and Anatomy, Drexel University College of Medicine, Philadelphia, Pennsylvania 19129, and ${ }^{2}$ Neurobiology Division, Shriver \\ Center, Waltham, Massachusetts 02452
}

\begin{abstract}
Microtubules in the axon are more resistant to severing by katanin than microtubules elsewhere in the neuron. We have hypothesized that this is because of the presence of tau on axonal microtubules. When katanin is overexpressed in fibroblasts, the microtubules are severed into short pieces, but this phenomenon is suppressed by the coexpression of tau. Protection against severing is also afforded by microtubule-associated protein 2 (MAP2), which has a tau-like microtubule-binding domain, but not by MAP1b, which has a different microtubule-binding domain. The microtubule-binding domain of tau is required for the protection, but within itself, provides less protection than the entire molecule. When tau (but not MAP2 or MAP1b) is experimentally depleted from neurons, the microtubules in the axon lose their characteristic resistance to katanin. These results, which validate our hypothesis, also suggest a potential explanation for why axonal microtubules deteriorate in neuropathies involving the dissociation of tau from the microtubules.
\end{abstract}

Key words: microtubule; katanin; tau; axon; neuron; MAP1b

\section{Introduction}

A growing body of evidence indicates that alterations in neuronal morphology are related to the capacity of the neuron to reconfigure its underlying microtubule scaffolding. Recent studies indicate that the capacity of a microtubule to move in a rapid and concerted manner is directly related to its length (Dent et al., 1999), with only the shortest microtubules displaying this kind of transport (Wang and Brown, 2002). Therefore, it follows that reconfiguration and transport of microtubules require that longer microtubules are severed into shorter pieces. Neurons are rich in a protein called katanin, which is an enzyme that hydrolyzes ATP to break the lattice of the microtubule. Katanin has been shown to be essential for severing microtubules from the centrosome of neurons (Ahmad et al., 1999) and to play key roles in generating short microtubules throughout all compartments of the neuron (Karabay et al., 2004; Yu et al., 2005).

Katanin consists of two subunits, one of which, termed P60katanin, is the enzyme that severs microtubules. The other subunit, termed P80-katanin, targets a portion of the katanin to the centrosome (McNally et al., 2000). Neurons contain high levels of katanin compared with other cell types, and these high levels

Received March 9, 2005; revised Feb. 3, 2006; accepted Feb. 6, 2006.

This work was supported by a National Institutes of Health grant and Tobacco Settlement Funds from the State of Pennsylvania to P.W.B. W.Y. was supported by a grant from the Edward Jekkal Foundation and Tobacco Settlement Funds from the State of Pennsylvania. We thank Shelley Halpain, Gloria Lee, Kenneth Kosik, Philip Gordon-Weeks, J. Chloe Bulinksi, and Itzhak Fischer for providing MAP constructs and antibodies for these studies. We thank Joanna Solowska, Arzu Karabay, and Sirin Korulu in our laboratory for helpful discussions and assistance.

*L.Q. and W.Y. contributed equally to this work.

Correspondence should be addressed to Dr. Peter W. Baas, Department of Neurobiology and Anatomy, Drexel University College of Medicine, 2900 West Queen Lane, Philadelphia, PA 19129. E-mail: pbaas@drexelmed.edu. DOI:10.1523/JNEUROSCI.5392-05.2006

Copyright $\odot 2006$ Society for Neuroscience $\quad$ 0270-6474/06/263120-10\$15.00/0 would theoretically sever the microtubules completely to subunits if not somehow attenuated by regulatory mechanisms (McNally and Vale, 1993). A potential clue as to how katanin is regulated derives from the observation that its severing activity is higher in mitotic extracts than in interphase extracts and that the higher activity is somehow related to phosphorylation (McNally et al., 2002). Interestingly, however, katanin itself appears not to be phosphorylated, suggesting that the phosphorylation of other proteins regulates katanin.

These observations support a model whereby katanininduced microtubule severing is regulated by microtubuleassociated proteins (MAPs), the phosphorylation of which causes them to dissociate from the microtubules (Baas and Qiang, 2005). This idea was originally prompted by the observation that the frog homolog of MAP4 diminishes the microtubule severing that occurs in response to katanin in vitro (McNally et al., 2002). In recent studies, we showed that brief bouts of P60-katanin overexpression in cultured neurons cause widespread severing and loss of microtubules from cell bodies, minor processes, and dendrites but not from the axon, where the microtubules appear to be far more resistant to severing (Yu et al., 2005). On this basis, we posited that axons may be enriched with a MAP that provides strong protection of microtubules against severing by katanin. Axons, at least from most central neurons, do not contain MAP4 (Dehmelt and Halpain, 2004) but are rich in tau. It is provocative to speculate that tau may play this protective role in the axon, given its tight regulation by phosphorylation (Johnson and Stoothoff, 2004), enrichment in the axon (Mandell and Banker, 1996), and the central roles it plays in neuropathies affecting axonal microtubules (Cairns et al., 2005; Stoothoff and Johnson, 2005). Here, we sought to determine whether tau is the chief protector of axonal microtubules from severing by katanin. 


\section{Materials and Methods}

Antibodies

Antibodies (Abs) included the following: rabbit anti-P60-katanin polyclonal Ab (1:1000 for immunostaining) (Karabay et al., 2004); rabbit anti-green fluorescent protein (anti-GFP) polyclonal Ab (1:500 for immunostaining; Abcam, Cambridge, UK); chicken polyclonal anti-myc tag $\mathrm{Ab}$ (1:500 for immunostaining; Abcam); mouse monoclonal anti-flag $\operatorname{tag} \mathrm{Ab}$ (1:500 for immunostaining; Sigma, St. Louis, MO); unconjugated mouse monoclonal anti- $\beta$-tubulin Ab (1:200 for immunostaining; Sig$\mathrm{ma})$; cyanine 3 (Cy3)-conjugated mouse monoclonal anti- $\beta$-tubulin $\mathrm{Ab}$ (1:300 for immunostaining; Sigma); mouse monoclonal anti-MAP2 Ab (1:200 for immunostaining and 1:1000 for Western blot; Chemicon, Temecula, CA); goat polyclonal anti-MAP1b Ab (1:200 for immunostaining; Santa Cruz Biotechnology, Santa Cruz, CA); rabbit polyclonal antiMAP1b Ab (1:500,000 for Western blot; provided by I. Fisher, Drexel University, Philadelphia, PA); rabbit polyclonal anti-MAP4 Ab (1:100 for immunostaining; also called LHB; provided by J. C. Bulinski, Columbia University, New York, NY); rabbit polyclonal anti-tau Abl (1:2000 for immunostaining in experiments of RFL6 cells; also called CR; provided by G. Lee, University of Iowa, Iowa City, IA); rabbit polyclonal anti-tau Ab2 (1:500 for immunostaining in experiments of rat neurons and 1:1000 for Western blot; also called DD8; provided by G. Lee); mouse monoclonal anti-glyceraldehyde-3-phosphate dehydrogenase (antiGAPDH; 1:500 for Western blot; Ambion, Austin, TX); and a series of appropriate fluorescent and HRP-conjugated secondary antibodies (Jackson ImmunoResearch, West Grove, PA).

\section{DNA constructs}

Constructs used for these studies were the following: pEGFP-C1 (control construct; BD Biosciences, Boston, MA); pEGFP-C1-p60 (rat P60katanin) (Karabay et al., 2004); pCMV/myc3b-P60 (rat P60-katanin); pMAP2c (human full-length MAP2c; provided by S. Halpain, The Scripps Research Institute, La Jolla, CA) (Gamblin et al., 1996); pMAP1b (mouse full-length; provided by P. R. Gordon-Weeks, King's College London, London, UK) (Gordon-Weeks and Fischer, 2000); pEGFP-C33R-tau (human three-repeat tau; provided by Dr. G. Lee) (Lee and Rook, 1992); pEGFP-C1-4R-tau (human four-repeat tau; provided by K. S. Kosik, University of California, Santa Barbara, CA) (Lu and Kosik, 2001); pRc/CMVflag123c (tauMTBD; C terminus of human tau with the microtubule-binding domain but without the projection domain; provided by G. Lee) (Leger et al., 1994); pRc/CMVflagn (tauMTNBD; N terminus of human tau without the microtubule-binding domain; provided by G. Lee) (Hall et al., 1997); pRc/CMVflagFFT/6d (tauFFT/6d, a natural human tau isoform that cannot bind to microtubules) (Luo et al., 2004); pEGFP-C1-MAP4 (human MAP4; provided by J. C. Bulinski) (Chang et al., 2001).

\section{Image acquisition and analysis}

Images were acquired with an AxioVert 200M microscope (Carl Zeiss, Oberkochen, Germany) coupled with an Orca-ER Digital CCD (Hamamatsu, Shizouka, Japan) and a $40 \times$ Plan-Neurofluar/1.3 or a $100 \times$ Plan-Neorofluar/1.3 numerical aperture objective. Images to be compared were taken at identical settings of exposure time, brightness, and contrast and analyzed with Axiovision 4.0 software. All measurements were taken as total fluorescence intensity per cell. Statistics were done using Student's $t$ test.

\section{Experiments on RFL6 fibroblasts}

Cell culture and transfection. RFL6 fibroblasts were transfected with MAP constructs and P60-katanin constructs using a Nucleofector (Amaxa, Gaithersburg, MD) with the manufacturer's program G-13. There were always four different groups in the studies with each MAP construct: (1) cells overexpressing one of the MAPs and GFP/myc (depending on which P60-katanin construct was used); (2) cells overexpressing P60-katanin and flag/GFP (depending on which MAP construct was used); (3) Cells overexpressing one of the MAPs and P60-katanin; (4) control group (cells overexpressing GFP/myc and/or flag/GFP). Fifteen micrograms of each plasmid and $10^{6}$ cells were used for each transfection. The cells were plated at a density of 8000 cells per well on glass coverslips mounted in the bottom of 35-mm-diameter Petri dishes with holes drilled in the bottom. Taxol was used at $5 \mu \mathrm{M}$. All of the cells were cultured for $12 \mathrm{~h}$ after transfection in the $\mathrm{CO}_{2}$ incubator before being fixed.

Immunostain analyses. For immunostaining of the expressed MAPs and/or P60-katanin, cultures were prepared as described previously (Karabay et al., 2004). To evaluate the protein level within each MAP overexpressed cell, the cells were fixed without previous extraction. The antibody to mouse MAP1b was used to compare the expressed MAP levels in RFL6 fibroblasts with the native levels of the MAP in rat hippocampal neurons by measuring the average fluorescence intensity in both cell types. Given that the antibody recognizes mouse and rat MAP1b about equally, this calculation was used as a baseline to achieve a level of expression approximately twice that in the neuron. Then, by comparing expression levels of the various MAPs that shared the same tag, we adjusted our transfection regime and selected fibroblasts that expressed generally similar levels of the other MAPs so that comparisons could be made among the different MAPs. Rabbit polyclonal anti-GFP Ab was used for tau3R and tau4R because they both are tagged with GFP. Rabbit polyclonal anti-tau Ab1 was then used to compare tau3R, tauFFT/6d, tau4R, and tauMTNBD because this Ab recognizes all four. Flag Ab was used to compare tauFFT/6d and tauMTBD because the tau Ab1 does not recognize tauMTBD. To quantify microtubule mass, RFL6 fibroblasts were simultaneously fixed and extracted to remove free tubulin as described previously (Yu et al., 2005). Two kinds of P60-katanin constructs were used: pCMV/myc3b-P60 and pEGFP-C1-P60. pCMV/myc3b-P60 was used when the cells were transfected with pEGFP-C3-3R-tau, pEGFP-C1-4R-tau, pEGFP-C1-MAP4. pEGFP-C1-P60 was used when the cells were transfected with pMAP2c, pMAP1b, pRc/CMVflag123c, $\mathrm{pRc} / \mathrm{CMV}$ flagn, and pRc/CMVflagFFT/6d. For the former, chicken polyclonal anti-myc tag $\mathrm{Ab}$ followed by $\mathrm{Cy} 3$-conjugated donkey anti-chicken IgG was used to reveal the overexpressed katanin; rabbit polyclonal antiGFP Ab followed by FITC-conjugated donkey anti-rabbit IgG was used to reveal the various MAPs; mouse monoclonal anti- $\beta$-tubulin $\mathrm{Ab}$ followed by Cy5-conjugated donkey anti-mouse IgG was used to reveal microtubules. For the latter, rabbit polyclonal anti-GFP followed by FITC-conjugated donkey anti-rabbit IgG was used to reveal P60-katanin; mouse monoclonal anti-MAP2 Ab followed by Cy5-conjugated donkey anti-mouse IgG was used to reveal MAP2c; goat polyclonal anti-MAP1b $\mathrm{Ab}$ followed by Cy5-conjugated donkey anti-goat IgG was used to reveal MAP1b; mouse monoclonal anti-flag tag Ab followed by Cy5-conjugated donkey anti-mouse IgG was used to reveal the flag-tagged MAPs. To immunostain microtubules in this latter group, we sequentially applied Cy3 directly conjugated anti- $\beta$-tubulin Ab. (So that samples could be compared against one another, controls were always stained with both the Cy3 and the Cy5 methods, and microscope settings were adjusted to equilibrate the signal.)

\section{Experiments on primary neurons}

Cell culture and transfection. Rat hippocampal neurons [cultured as in $\mathrm{Yu}$ and Baas (1994)] were transfected with small interfering RNA (siRNA) for MAP2, tau, or MAP1b using a Nucleofector (Amaxa) with the manufacturer's program G-13. For MAP2 siRNA, we used a sequence previously reported to be effective (Krichevsky and Kosik, 2002). For tau and MAP1b, we used a mixture of four different siRNA duplexes designed against different regions of each molecule using Dharmacon (Lafayette, CO) custom SMARTpool siRNA service. The Dharmacon accession number for rat MAP2 is NM_013066; for rat MAP1b is XM_215469, and for rat tau is NM_017212. The nonspecific duplex III (Dharmacon) was used as control. siRNA was dissolved to $200 \mu \mathrm{M}$, aliquoted, and stored at $-20^{\circ} \mathrm{C}$. siRNA concentration at transfection was $18 \mu \mathrm{m}$. The siRNA treated neurons were cultured for $48 \mathrm{~h}$ in plastic dishes coated with poly-L-lysine before being replated. All of the siRNA treated neurons were kept for 1,3 , or $5 \mathrm{~d}$, respectively before being fixed for the detection of different MAP levels.

Two days later, in each group, some of neurons were replated at a density of 8000 cells per well on glass coverslips mounted in the bottom of the drilled 35-mm-diameter Petri dishes. The rest of them were maintained in the plastic dishes. The transfections of pEGFP-C1-P60 were performed $2 \mathrm{~d}$ after the replating. For transfections of P60-katanin, we used Lipofectamine 2000 (11668-027; Invitrogen, San Diego, CA). Two 

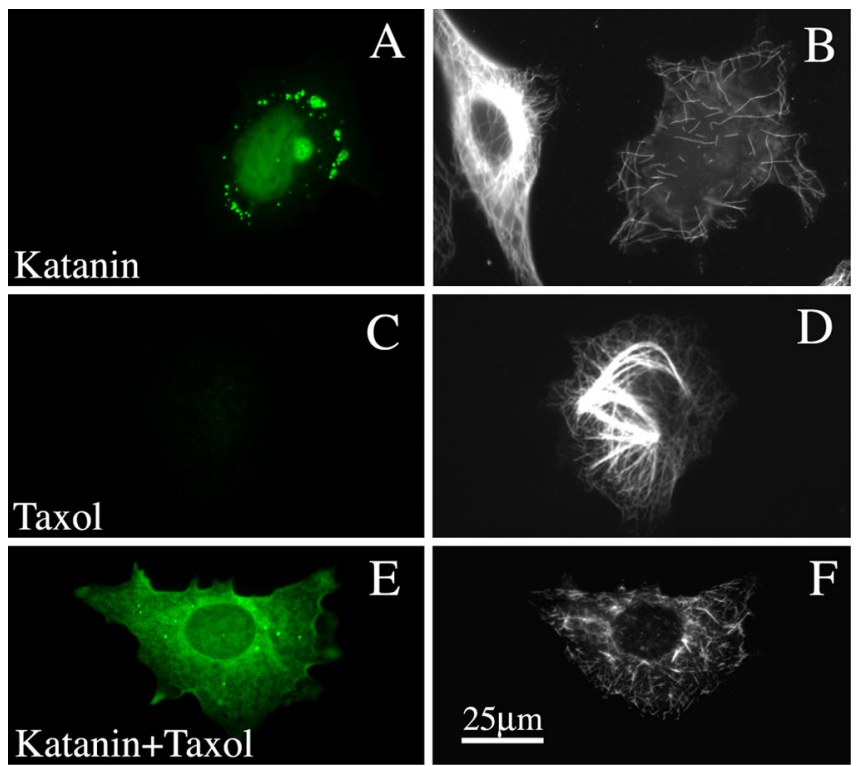

Figure 1. Microtubules are not protected from P60-katanin-induced severing by Taxol. $\boldsymbol{A}$ and $\boldsymbol{E}$ show expression of GFP-P60-katanin (in green), whereas $\boldsymbol{B}, \boldsymbol{D}$, and $\boldsymbol{F}$ show immunostains for microtubules. $A, B$, Two cells, only one of which is expressing GFP-P60-katanin. The nonexpresser shows a normal dense splayed microtubule array, whereas the expresser shows far less microtubule mass and only a scattering of very short microtubules. C, D, A cell not expressing GFP-P60-katanin but treated with Taxol. A portion of the microtubules appears as thick bundles. $E, F$, A cell expressing GFP-P60-katanin in the presence of Taxol. Note that the microtubule mass is severely reduced, and both bundled and unbundled microtubules are very short. Scale bar: (in $\boldsymbol{F}) \boldsymbol{A}-\boldsymbol{F}, 30 \mu \mathrm{m}$.

to four micrograms of DNA constructs and 5-10 $\mu \mathrm{g}$ of Lipofectamine 2000 were used per $35 \mathrm{~mm}$ dish. The ratio of DNA and Lipofectamine 2000 was 1:2.5. The cells were incubated in the DNA/Lipofectaminecontaining medium for $5 \mathrm{~h}$. After transfection, neurons were transferred to $37^{\circ} \mathrm{C}$ hippocampal neuron plating medium (Neurobasal medium supplemented with $2 \%$ B27, 0.3\% glucose, $1 \mathrm{~mm}$ glutamine, and 5\% FBS). Twelve hours later, the cells replated on $35 \mathrm{~mm}$ dishes were fixed for immunostaining, and the cells replated on plastic dishes were collected for Western blots for the three MAPs. The levels of MAPs were detected by immunostaining after fixation each day after the replating procedure.

Immunostain analyses. Mouse monoclonal anti-MAP2 Ab, rabbit polyclonal anti-tau $\mathrm{Ab}$, and goat polyclonal anti-MAP1b Ab were used to detect the three MAPs, respectively, in the siRNA-treated hippocampal neurons. GFP antibodies were applied to find the P60-kataninoverexpressing neurons. To quantify microtubule mass, neurons were simultaneously fixed and extracted to remove free tubulin as described previously (Yu et al., 2005). To immunostain microtubules, we applied $\mathrm{Cy} 3$ directly conjugated anti- $\beta$-tubulin $\mathrm{Ab}$.

Western blot analyses. Western blot analyses were performed according to established procedures (Sambrook et al., 1989). Neurons cultured in the plastic dishes were suspended with cold $1 \times$ PBS, lysed in the sample buffer with SDS, and then the proteins were resolved in 10 or $7.5 \%$ SDS-PAGE gels ( $10 \%$ gel was used in the Western blots to detect the level of MAP2c and Tau, whereas 7.5\% gel was used in the Western blots to detect the level of MAP2a/b and MAP1b), and transferred onto nitrocellulose membranes $\left(300 \mathrm{~mA}\right.$; overnight at $\left.4^{\circ} \mathrm{C}\right)$. Transfers were probed with MAP2 antibody against both MAP2a/b and MAP2c, with MAP1b antibody (provided by I. Fisher) and with Tau antibody (DD8), respectively. The corresponding secondary HRP-conjugated antibody was used. After reaction with chemiluminescent peroxidase substrate (Super Signal; Pierce, Rockford, IL), each blot was covered with x-ray film. For each blot, we used three different exposure times (5, 15, 30 s). Films were imaged using an Epson (Long Beach, CA) Perfection 1280 scanner. Protein markers were used to indicate the appropriate molecular weights. GAPDH was used as the internal control in each group to show that the same amount of protein was loaded in each group.
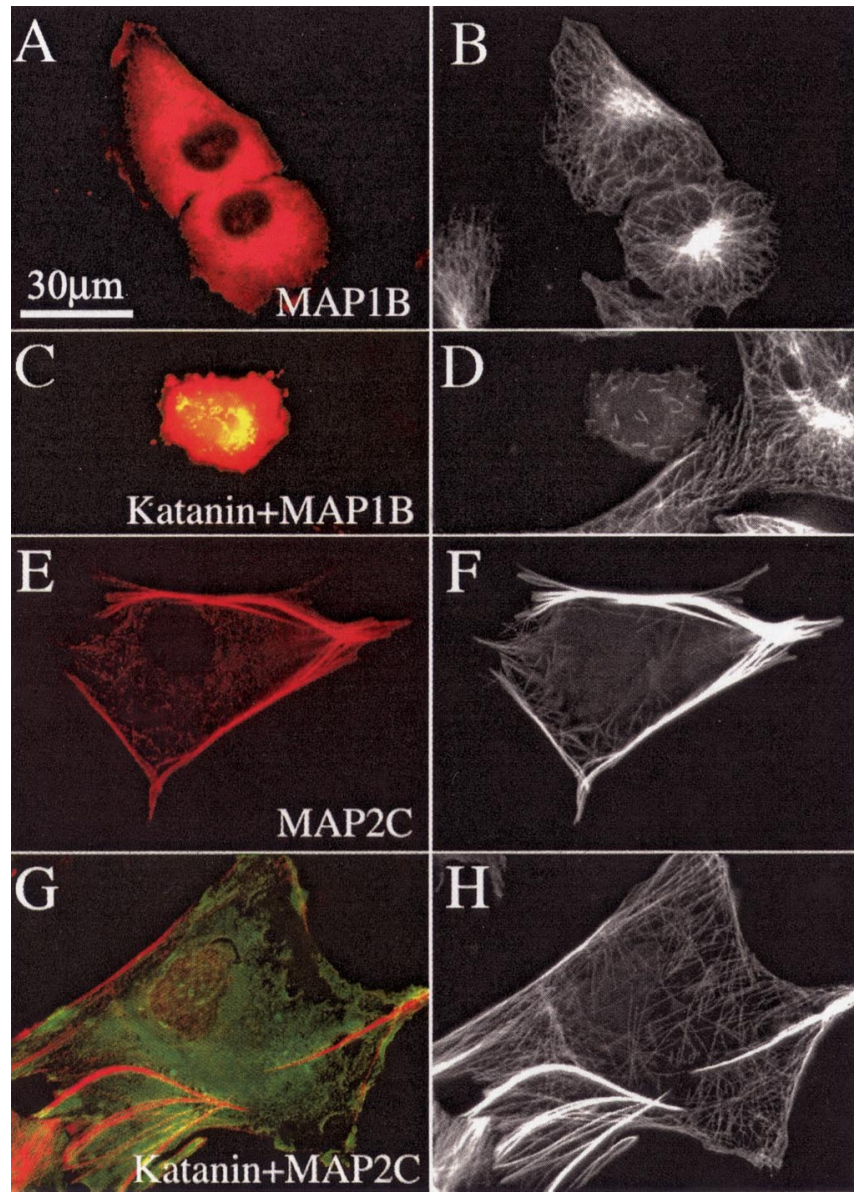

Figure 2. MAP2C protects microtubules from being severed by P60-katanin, but MAP1b does not. $\boldsymbol{A}, \boldsymbol{C}, \boldsymbol{E}, \mathbf{G}, \mathrm{GFP}-\mathrm{P} 60$-katanin in green and immunostain for either plasmid-expressed MAP2 or MAP1b in red. $\boldsymbol{B}, \boldsymbol{D}, \boldsymbol{F}, \boldsymbol{H}$, Immunostains for microtubules. $\boldsymbol{A}, \boldsymbol{B}$, Cells that are not overexpressing P60-katanin. As shown in $\boldsymbol{A}$ and $\boldsymbol{B}$, MAP1b expression does not cause abnormal bundling of microtubules. As shown in $\boldsymbol{C}$ and $\boldsymbol{D}$, a cell overexpressing MAP1b and P60-katanin shows only a scattering of very short microtubules and severely reduced microtubule levels. (A neighboring cell not expressing MAP1b or P60-katanin displays a normal microtubule array). As shown in $\boldsymbol{E}$ and $\boldsymbol{F}$, MAP2c expression causes the formation of dense bundles of microtubules. As shown in $\boldsymbol{G}$ and $\boldsymbol{H}$, the microtubules in MAP2c-expressing cells show no indication of severing by overexpression of P60-katanin, and the microtubule mass is not reduced. Scale bar: $\boldsymbol{A}-\boldsymbol{H}, 30 \mu \mathrm{m}$.

\section{Results}

There are two sets of studies presented here, one on RFL6 fibroblasts and the other on primary cultured neurons. The studies on RFL6 fibroblasts seek to test, in a relatively simple non-neuronal cell, whether individual neuronal MAPs have the capacity to protect microtubules from severing by overexpressed P60-katanin. These studies also include experiments using Taxol, which stabilizes and bundles microtubules by a mechanism entirely different from MAPs. In the second set of studies, we take the inverse approach of knocking down individual MAPs from cultured hippocampal neurons to determine whether the microtubules in the axon become any less resistant to severing by katanin. The RFL6 cells and primary neurons are both from rat.

\section{Stabilization and bundling by Taxol does not protect microtubules from severing}

In a first set of studies on the RFL6 fibroblasts, we investigated whether stabilization and bundling of microtubules by Taxol alters their response to P60-katanin overexpression. We chose 

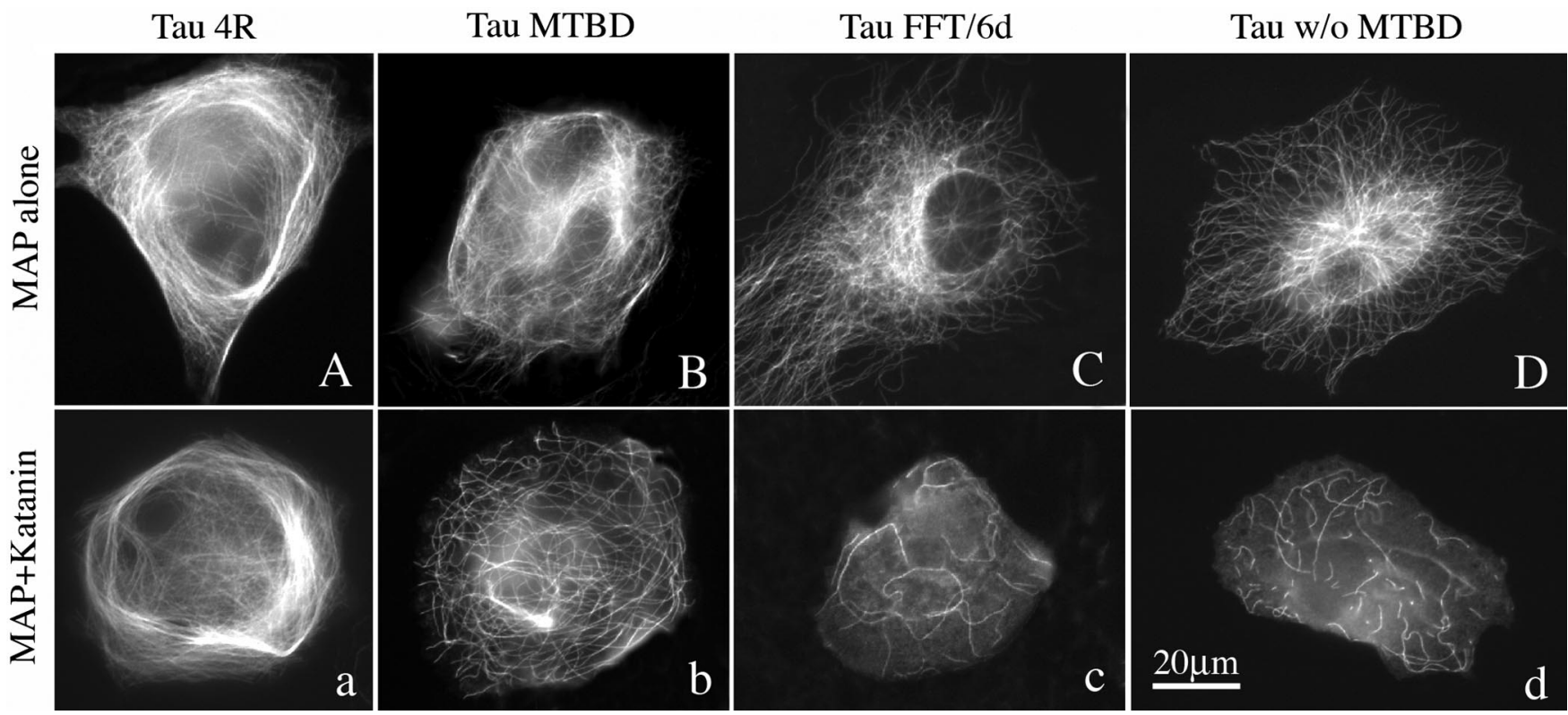

Figure 3. Results obtained with various tau constructs expressed together with P60-katanin. Cells in the top row were transfected with the tau constructs alone. Cells in the bottom row were transfected with the tau constructs together with P60-katanin. Four-repeat tau generates microtubule bundling $(\boldsymbol{A})$ and no indication of microtubule severing $(\boldsymbol{a})$. The microtubule-binding domain alone generates some bundling, but not as much as the four-repeat isoform $(\boldsymbol{B})$, and provides some protection against severing $(\boldsymbol{b})$, but not as much as the four-repeat or the three-repeat (not shown in figure) full-length versions of tau. Two versions of tau that lack the microtubule-binding domain, one naturally occurring $(\boldsymbol{C}, \boldsymbol{c})$ and one generated by experimental truncation $(\boldsymbol{D}, \boldsymbol{d})$, show no protection against severing. w/0, Without. Scale bar: (in d) $30 \mu \mathrm{m}$.

RFL6 fibroblasts for these studies because they are very flat, and hence excellent for imaging microtubules using immunofluorescence, and because MAP4 is the only traditional fibrous MAP endogenously expressed. The levels of MAP4 in these cells, revealed by immunostaining, are generally similar to the levels in other fibroblastic cells used for microtubule-related studies over the years (data not shown). No other traditional fibrous MAP is endogenously expressed in these cells. For the present studies, we used pEGFP-C1 (control construct) or pEGFP-C1-P60 (rat P60katanin) (Karabay et al., 2004). When P60-katanin was overexpressed in RFL6 fibroblasts (Fig. 1A), the microtubules no longer appeared as a dense array of long polymers extending from cell center to cell periphery, but rather appeared as a scattering of short polymers, generally only a few micrometers in length (Fig. $1 \mathrm{~B}$ ). There was also a marked diminution in polymer levels (to $63.5 \%$ of control levels; $p<0.01$ ). Quantitative analyses using an $\mathrm{Ab}$ against rat $\mathrm{P} 60$-katanin indicated that the high expressers generally expressed no more than 39\% higher levels of P60katanin than control RFL6 fibroblasts. When the cells were treated with Taxol (without the expression of P60-katanin) (Fig. $1 C$ ), free tubulin was almost completely driven into polymer, and the microtubules formed dense bundles (Fig. 1D). Taxol treatment increased the polymer levels above controls by $17.4 \%$ ( $p<$ 0.05 ). However, the expression of P60-katanin (Fig. 1E) nevertheless caused the microtubules to break into short pieces (Fig. $1 F$ ), indicating that the stabilization and bundling of microtubules per se does not protect them from katanin-induced severing. Polymer levels were down by $65.0 \%(p<0.01)$ when P60katanin was overexpressed in Taxol-treated cells. Figure 4 shows the quantitative data. These results are consistent with the results of studies in vitro showing that katanin effectively severs Taxolstabilized microtubules (Vale, 1991).

\section{Certain MAPs protect microtubules from severing}

To test the capacity of MAPs to protect the microtubules against severing, we first overexpressed MAP2c, MAP1b, or MAP4. The
DNA constructs used for these studies were: pEGFP-C1 (control construct); pEGFP-C1-P60 (rat P60-katanin); pCMV/myc3bP60 (rat P60-katanin); pMAP2c (human MAP2c) (Gamblin et al., 1996), pMAP1b (mouse MAP1b) (Gordon-Weeks and Fischer, 2000); pEGFP-C1-MAP4 (human MAP4) (Chang et al., 2001). To compare the results of the different MAPs against one another, we achieved approximately equivalent levels of expression of all of the MAPs. As a baseline, we used MAP1b, because the construct is from the mouse sequence and hence could be compared more accurately than the other constructs against endogenous levels in cultured rodent neurons. The transfection protocol was optimized to achieve levels approximately twice the endogenous levels in the cell body of cultured hippocampal neurons (as revealed by immunostaining; data not shown). Then, we achieved approximately the same expression levels of the other MAP constructs by optimizing the conditions of the transfection protocol and chose cells that most closely matched the expression level achieved for MAP1b. With regard to P60-katanin, we selected the most highly expressing cells for our analyses, because they gave the most consistent results.

Figure $2 A-D$ shows the results obtained with MAP1b. MAP1b expression, shown in Figure 2, $A$ and $B$, did not cause microtubules to bundle, which was expected on the basis of previous studies (Takemura et al., 1992). No significant changes were detected in polymer levels in response to MAP1b $(p>0.05)$. As shown in Figure 2, $C$ and $D$, the microtubules were severed into small pieces in cells overexpressing MAP $1 \mathrm{~b}$ and $\mathrm{P} 60$-katanin. The degree of severing and polymer loss $(61.1 \%$; $p<0.01)$ was similar to controls overexpressing P60-katanin alone. Figure 4 shows the quantitative data. A potential concern with these studies was the degree to which the ectopically expressed MAP1b actually associates with microtubules, given the diffuse appearance of the fluorescence signal, and the lack of bundling induced by this MAP. However, previous studies indicate that ectopically expressed MAP1b, while not bundling microtubules, clearly stabilizes them against disassembly (as assessed by nocodazole sensitivity) and 
slows their dynamics as indicated by enhanced acetylation (Takemura et al., 1992). Thus, at least some of the ectopically expressed MAP1b associates with microtubules. We found this also to be the case in our studies (data not shown), but even so, we wished to be particularly careful about this result, so we overexpressed the MAP1b construct at levels several times the endogenous levels found in neurons. Even at these very high levels, no evidence for protection against P60-katanin-induced severed was observed.

Figure $2 E-H$ shows the results with MAP2c. We chose to use MAP2c rather than the larger adult MAP2 because the former is present in developing axons (whereas the latter is more relevant to dendrites) and because it expresses more reliably (Meichsner et al., 1993). Figure 2, $E$ and $F$, shows marked bundling of microtubules by MAP2c, although there were also numerous unbundled microtubules as well. Polymer levels were increased by $28.0 \%$ $(p<0.05)$ in the presence of MAP2c. Overexpression of P60katanin did not alter the length of the microtubules, their bundling, or the levels of polymer in any detectable way (Fig. $2 E-H$ ). Unbundled microtubules remained long, indicating that it was not the bundling per se that impeded severing. There was no significant loss of microtubule mass from either group $(p>$ 0.05). Figure 4 shows the quantitative data.

A key difference between MAP2c and MAP1b is that they have very different microtubule-binding domains. However, the microtubule-binding domain of MAP2c is very similar to that of tau and MAP4 (Seitz et al., 2002), suggesting that tau and MAP4 may be similar to MAP2c with regard to their capacity to protect the microtubule against severing. Also shown in Figure 4 are the quantitative data for results obtained with MAP4; no significant increase was detected in the microtubule mass above control levels $(p>0.05)$. When P60-katanin was overexpressed with MAP4, microtubule levels were diminished by $33.3 \%(p<0.05)$. As with the MAP2c studies, unbundled microtubules were protected against severing as well as bundled microtubules. Thus, MAP4 provides protection against P60-katanin, but not to the same degree as MAP2c.

\section{Studies on tau indicate protective properties require the microtubule-binding domain but are assisted by the projection domain}

In a final set of studies on the fibroblasts, we used several different tau constructs and examined their capacity to alter the response of the fibroblasts to P60-katanin overexpression. For these studies, we used pEGFP-C1 (control construct); pEGFP-C1-P60 (rat P60-katanin); pCMV/myc3b-P60 (rat P60-katanin); pEGFP-C33R-tau (human three-repeat tau) (Lee and Rook, 1992); pEGFPC1-4R-tau (human four-repeat tau) (Lu and Kosik, 2001); pRc/ CMVflag123c (tauMTBD; C terminus of human tau with the microtubule-binding domain but without the projection domain) (Leger et al., 1994); pRc/CMVflagn (tauMTNBD; N terminus of human tau without the microtubule-binding domain) (Hall et al., 1997); and pRc/CMVflagFFT/6d (tauFFT/6d; a natural human tau isoform that cannot bind to microtubules) (Luo et al., 2004).

Figure 3 shows P60-katanin-overexpressing cells also expressing various tau constructs. Tau3R is not shown. Figure $3, A$ and $a$, is Tau4R. Figure 3, $B$ and $b$, is Tau-MTBD. Figure $3, C$ and $c$, is Tau-MTNBD. Figure 3, $D$ and $d$, is Tau-FFT/6d. Tau4R increased microtubule mass by $20.8 \%$ above control levels $(p<0.05)$; coexpression with $\mathrm{P} 60$-katanin produced no diminution in microtubule mass $(p>0.05)$. Tau3R increased microtubule mass by $5.1 \%$ above control levels $(p<0.05)$; coexpression with P60katanin reduced the microtubule mass by $25.5 \%(p<0.05)$,
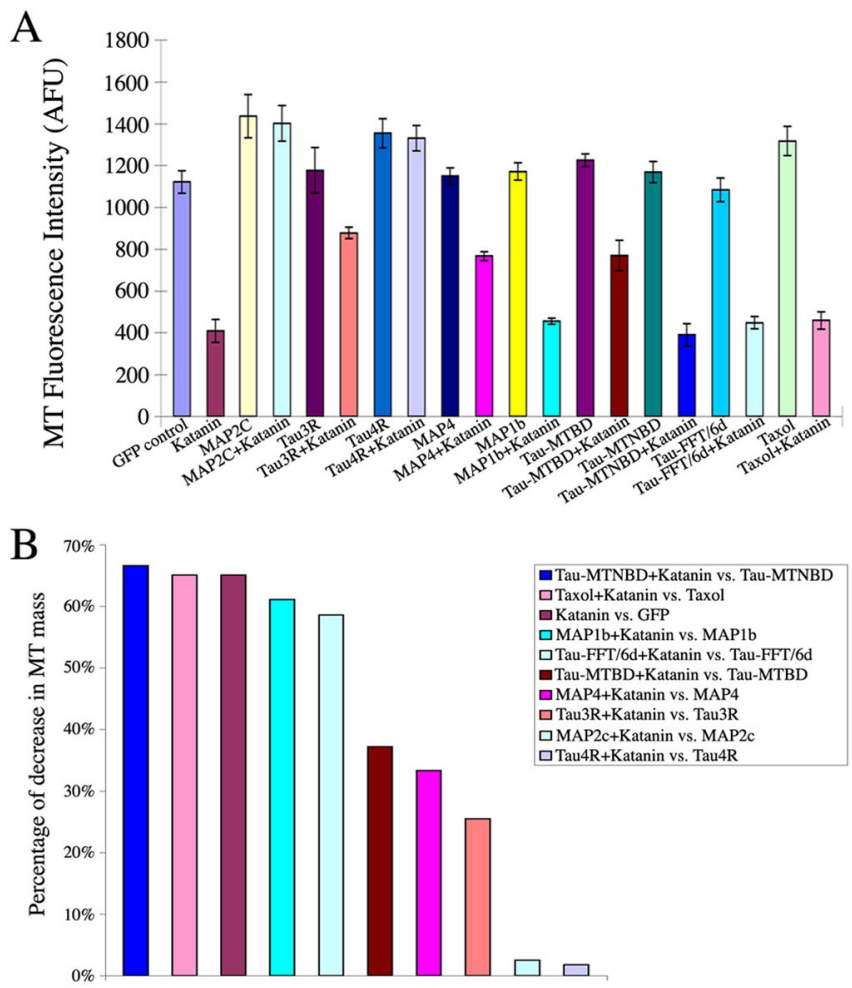

Figure 4. Quantitative analyses of microtubule mass in fibroblasts induced to overexpress katanin together with various MAP constructs. $\boldsymbol{A}$, The quantitative data graphically on the average fluorescence intensity of microtubule total mass. GFP (GFP alone as the control); katanin (P60-katanin alone); Taxol (control cells cultured in Taxol-containing medium); Taxol plus katanin (P60-katanin transfected cells cultured in Taxol-containing medium); MAP2C (MAP2C alone); MAP2c plus katanin (the cells expressing MAP2c and P60-katanin); MAP1b (MAP1b alone); MAP1b plus katanin (cells expressing MAP1b and P60-katanin); MAP4 (MAP4 alone); MAP4 plus katanin (cells expressing MAP4 and P60-katanin); tau3R (tau-3R alone); tau3R plus katanin (cells expressing tau3R and P60-katanin); tau4R (cells expressing tau4R alone); tau4R plus P60-katanin (cells expressing tau4R and P60-katanin); tauMTBD (tauMTBD alone); tauMTBD plus katanin (cells expressing tauMTBD and P60-katanin); tauMTNBD (tauMTNBD alone); tauMTNBD plus katanin (cells expressing tauMTNBD and P60-katanin); tauFFT/6d (tauFFT/6d alone); tauFFT/6d plus katanin (cells expressing tauFFT/6d and P60-katanin). B, Bar graph showing calculations on the percentage diminution in microtubule mass in cells with the various treatments (MAP expression, Taxol, GFP alone) as a result of expression of P60-katanin, from high to low. Error bars represent SE. MT, Microtubule; AFU, arbitrary fluorescence units.

indicating that Tau3R protects the microtubules against P60katanin, but not as well as MAP2c or Tau4R. Tau-MTBD increased microtubule mass by $9.2 \%$ above control levels $(p<$ $0.05)$; coexpression with P60-katanin reduced the microtubule mass by $37.2 \%(p<0.05)$, indicating that the microtubulebinding domain alone protects the microtubules against P60katanin, but not as well as MAP2c or Tau4R and not even as well as Tau3R. The two tau isoforms that lacked the microtubulebinding domain resulted in no increase in microtubule mass; expression of P60-katanin diminished the microtubule mass by 58.6 and $66.6 \%$, which were statistically indistinguishable from the diminution obtained with cells overexpressing P60-katanin but not expressing any tau constructs $(p>0.05)$. Thus, the microtubule-binding domain is essential for the protection but does not, within itself, provide as much protection as the intact molecule. As with the MAP2c and MAP4 studies, unbundled microtubules were protected against severing as well as bundled microtubules. Quantitative data are shown in Figure 4. 


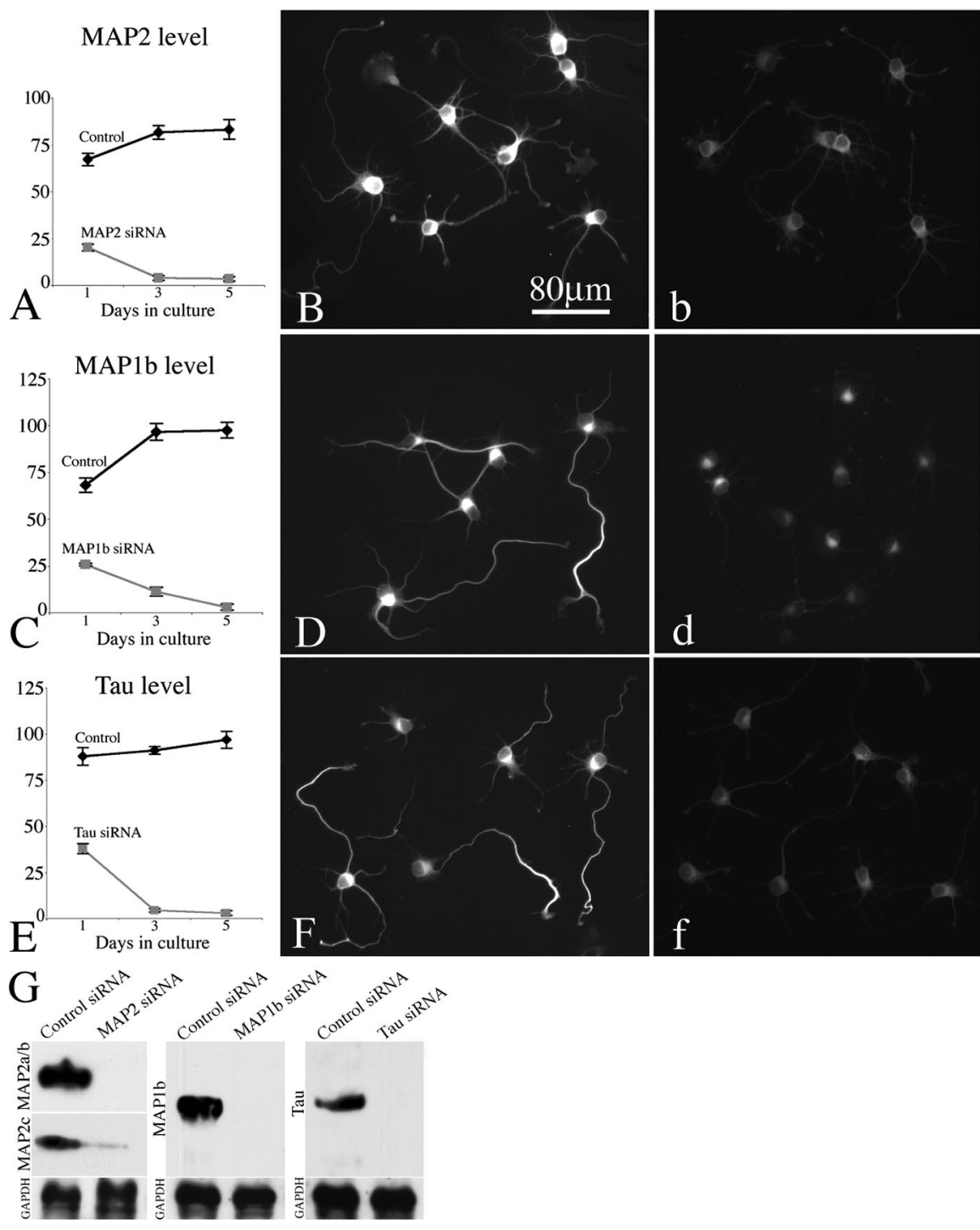

Figure 5. Quantitative analysis of MAP levels after treatment with siRNAs. Control siRNA and siRNAs to MAP2, MAP1b, and tau were transfected into hippocampal neurons. Cultures were fixed 1, 3, and $5 \mathrm{~d}$, respectively, after transfection and immunostained for each MAP to evaluate its level as a result of the siRNA. Immunofluorescence indicates depletion of MAP2, MAP1b, and tau in siRNA-treated neurons $(\boldsymbol{b}, \boldsymbol{d}, \boldsymbol{f})$, compared with control siRNA-treated neurons $(\boldsymbol{B}, \boldsymbol{D}, \boldsymbol{F})$, is shown. $\boldsymbol{A}, \boldsymbol{C}, \boldsymbol{E}$, The quantification of total fluorescence intensity within the soma revealing the progressive loss of MAP2, MAP1b, and tau depletion by siRNA. By day 5 , the loss of MAP2, MAP1b, and tau in siRNA treated neurons are 95, 99, and 99\%, respectively. $\mathbf{G}$, Western blot of whole-cell extracts probed with the MAP2, MAP1b, and tau Ab, confirming the protein-lowering effect. GAPDH was used as the internal control. Error bars represent SE.

\section{Depletion of MAPs from cultured hippocampal neurons by siRNA}

Over the years, a variety of different methods have been used to deplete MAPs from neurons. A great deal of work was done in the early 1990s using antisense oligonucleotides to deplete MAPs from cultured cerebellar macro-neurons, which are quite similar to cultured hippocampal neurons. Both types of neurons undergo a series of developmental stages in which the neuron first extends a broad lamellipodium (stage I) that coalesces into several essentially identical minor processes (stage II), after which one of the minor processes elongates rapidly and becomes the axon (stage III). The studies using antisense oligonucleotides suggested that MAP2 was required for the formation of the minor processes (Caceres et al., 1992), whereas tau and MAP1b are important for axonal differentiation (Caceres and Kosik, 1990; Cac- eres et al., 1991; Brugg et al., 1993; DiTella et al., 1996). In our studies, we used siRNA, which was introduced by nucleofection at the time of plating (He et al., 2005). Tau and MAP1b were depleted by using combinations of four siRNA sequences, whereas MAP2 was depleted using a sequence reported previously (see Materials and Methods). After $2 \mathrm{~d}$ in culture, most of the MAP protein was depleted (as assessed by quantitative immunostains and Western blots) (Fig. 5), at which time the neurons were triturated and replated so that we could observe the capacity of the neuron to differentiation an axon after the MAP protein had been depleted. The protein level of MAPs remained low after the replating and was determined to be $95-99 \%$ depleted by the fifth total day (3 d after replating) (Fig. $5 G)$. Unlike previous studies using antisense oligonucleotides, we observed no major phenotypic deficiencies in these neurons as they underwent development after depletion of tau, MAP2, or MAP1b. Lamellipodia, minor processes, and axons all developed and appeared morphologically normal. However, we did note that the timetable for axonal differentiation was slower in neurons depleted of MAP1b (but not neurons depleted of tau or MAP2) compared with control neurons, with only $51 \%$ in stage III on the third day after replating as opposed to $77 \%$ for controls. In addition, axons were somewhat shorter after depletion of tau (14\% shorter compared with controls) or MAP2 (14\% shorter compared with controls) and shorter yet in neurons depleted of MAP1b ( $24 \%$ shorter compared with controls). There was no difference in the lengths of the minor processes with any of the MAP depletions. Microtubule levels in the cell bodies were down by $13 \%$ in neurons depleted of MAP1b but were unchanged in neurons depleted of tau or MAP2. These data are shown in detail with statistical analyses in Figure 6 and its legend. Interestingly, in the axons of about one-third of the neurons depleted of MAP1b (but not MAP2 or tau), we observed a difference in the distribution (but not total levels) of microtubules; microtubules were denser in the distal half of the axon and sparser in the proximal half, compared with controls.

These results add to those obtained from a variety of studies using different techniques to knock out, knock down, or inhibit MAP functions in neurons, some of which show dramatic phenotypes and some of which do not (Tint et al., 1998; Dawson et al., 2001; Teng et al., 2001; Gonzalez-Billault et al., 2002; Harada et al., 2002). It is unclear why the phenotypes vary in these studies, but it may have to do with compensatory mechanisms that are enabled under different conditions. The antisense experiments of the 1990s are most similar to ours but produced very different results. Although the reasons for this are unknown, we have more 
confidence in the siRNA method, which has been shown to be more specific and less problematic than the older antisense approach (Rossi and Eckstein, 2003).

\section{Overexpression of P60-katanin in hippocampal neurons depleted of individual MAPs}

We next performed an experiment in which P60-katanin was overexpressed for $12 \mathrm{~h}$ in these experimental neurons on the third day after replating. We used the same protocol as in our previous study (Yu et al., 2005), in which the levels of P60 were elevated $\sim 39 \%$ by the end of the $12 \mathrm{~h}$ time frame of expression. We performed this experiment in neurons treated with control siRNA (Fig. $7 A, B, a, b$ ) or siRNA for MAP1b (Fig. $7 C, D, c, d$ ), MAP2 (data not shown), or tau (Fig. $7 E, F, e, f$ ). (In Fig. 7, panels with capital letters are the original immunofluorescence images, whereas panels with small letters show pseudocolored regions of axons to better illustrate fluorescence intensity.) As reported previously, the microtubule levels were substantially reduced in the cell bodies $(36 \%$ diminution) and minor processes $(65 \%$ diminution) but not in the axons of the control neurons after P60-katanin overexpression. The same result was obtained in the case of neurons depleted of MAP1b and MAP2. However, in the case of neurons depleted of tau, the overexpression of P60-katanin resulted in a substantial loss of microtubule mass from the axon $(63 \%$ diminution). These data, which are shown quantitatively in Figure $7 G$, indicate a unique role for tau in the protection of axonal microtubules against severing by katanin.

\section{Discussion}

Here, we sought to test a model whereby the association of certain MAPs with axonal microtubules modulates the capacity of katanin to sever them (Baas and Qiang, 2005). According to this model, dissociation of the MAP from the microtubule, presumably by local phosphorylation of the MAP, allows katanin to access the microtubule lattice. This model is appealing because it can explain how the neuron maintains a population of very long microtubules while also generating short microtubules via tightly regulated severing events. For example, the local phosphorylation of the MAP within a discrete region of the axon could result in the formation of a collateral branch by allowing microtubules to be locally severed in that region (Baas and Qiang, 2005). Our studies focused on tau, MAP1b, and the juvenile variant of MAP2 termed MAP2c, because all three are present in developing axons (Brugg et al., 1993; Meichsner et al., 1993; Mandell and Banker, 1996). We also included MAP4, which is present in non-neuronal cells and some types of neurons (Chapin et al., 1995).

We found that tau, MAP2c, and MAP4 provide strong protection of the microtubules against severing by overexpressed P60katanin. All three of these MAPs share a similar microtubulebinding domain (Chapin and Bulinski, 1991; Dehmelt and Halpain, 2004). The level of protection relates to the binding affinity of the MAP to the microtubule, given that MAP2c and the rescence units.
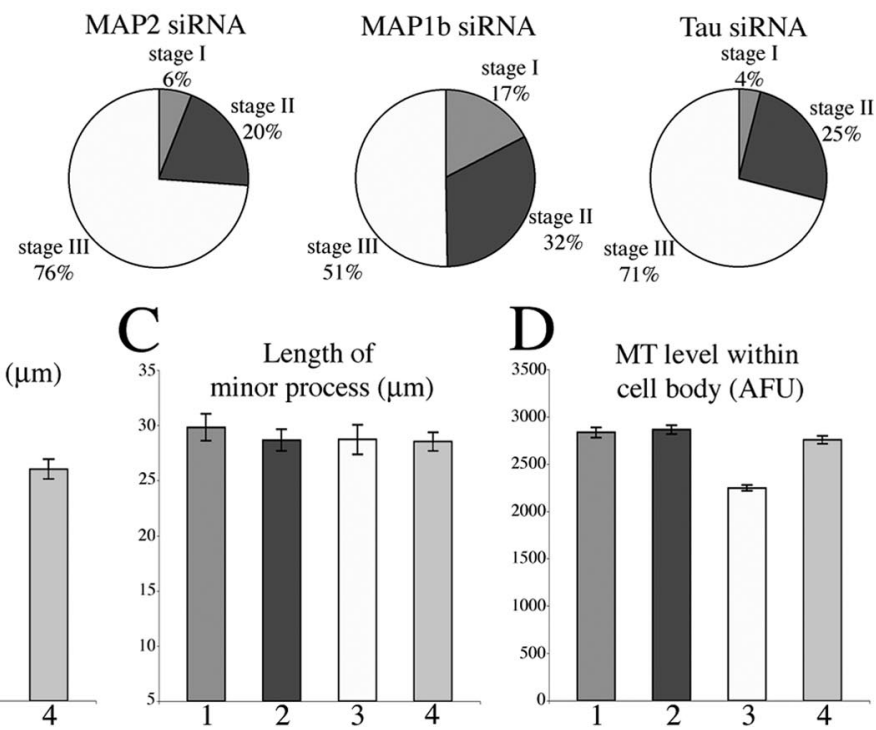
Length of axon $(\mu \mathrm{m})$
Figure 6. Effects of depleting MAP2, MAP1b, and tau on the development of hippocampal neurons in culture. Hippocampal
neurons were divided into four groups, treated with control siRNA or siRNA to MAP2, MAP1b, or tau, respectively. Replating of quantive analysis of the lengths of minor processes and axons of neurons in each group. Compared with the control, the axonal 作 MAP1b siRNA-treated, and tau siRNA-treated group, respectively. Error bars represent SE. MT, Microtubule; AFU, arbitrary fluo-

four-repeat form of tau protect somewhat better and have stronger binding affinities to the microtubule than the three-repeat form of tau (Seitz et al., 2002). We found that the microtubulebinding domain of tau provides some protection against microtubule severing by P60-katanin, but not to the degree of the entire molecule. However, without the binding domain, the rest of the molecule provides no protection whatsoever. Thus, the likely mechanism by which MAPs regulate microtubule severing is to physically restrict access of the severing proteins to the microtubule rather than interacting directly with the severing proteins. We speculate that the projection domain provides additional steric hindrance of the access of katanin to the microtubule.

In contrast with the tau/MAP2/MAP4 family of MAPs, we found that MAP1b provides no protection against katanininduced microtubule severing, nor does Taxol. Both of these molecules stabilize the microtubules against disassembly. Taxol bundles microtubules but MAP1b does not. We do not believe that the bundling of the microtubules is important to the protection phenomenon because Taxol-induced bundles are very sensitive to severing and because the unbundled microtubules in cells overexpressing tau, MAP2, or MAP4 were also protected against severing. In contrast with the other expressed MAPs, MAP1b did not cause microtubules to bundle and displayed a more diffuse nonfilamentous appearance, suggesting that not all of the protein associated with the microtubules. This may be because of the phosphorylation state of the expressed protein (Goold and Gordon-Weeks, 2005; Kawauchi et al., 2005). For this reason, we also examined cells that were overexpressing severalfold higher levels of MAP1b. There was still no detectable protection against microtubule severing (although the microtubules 


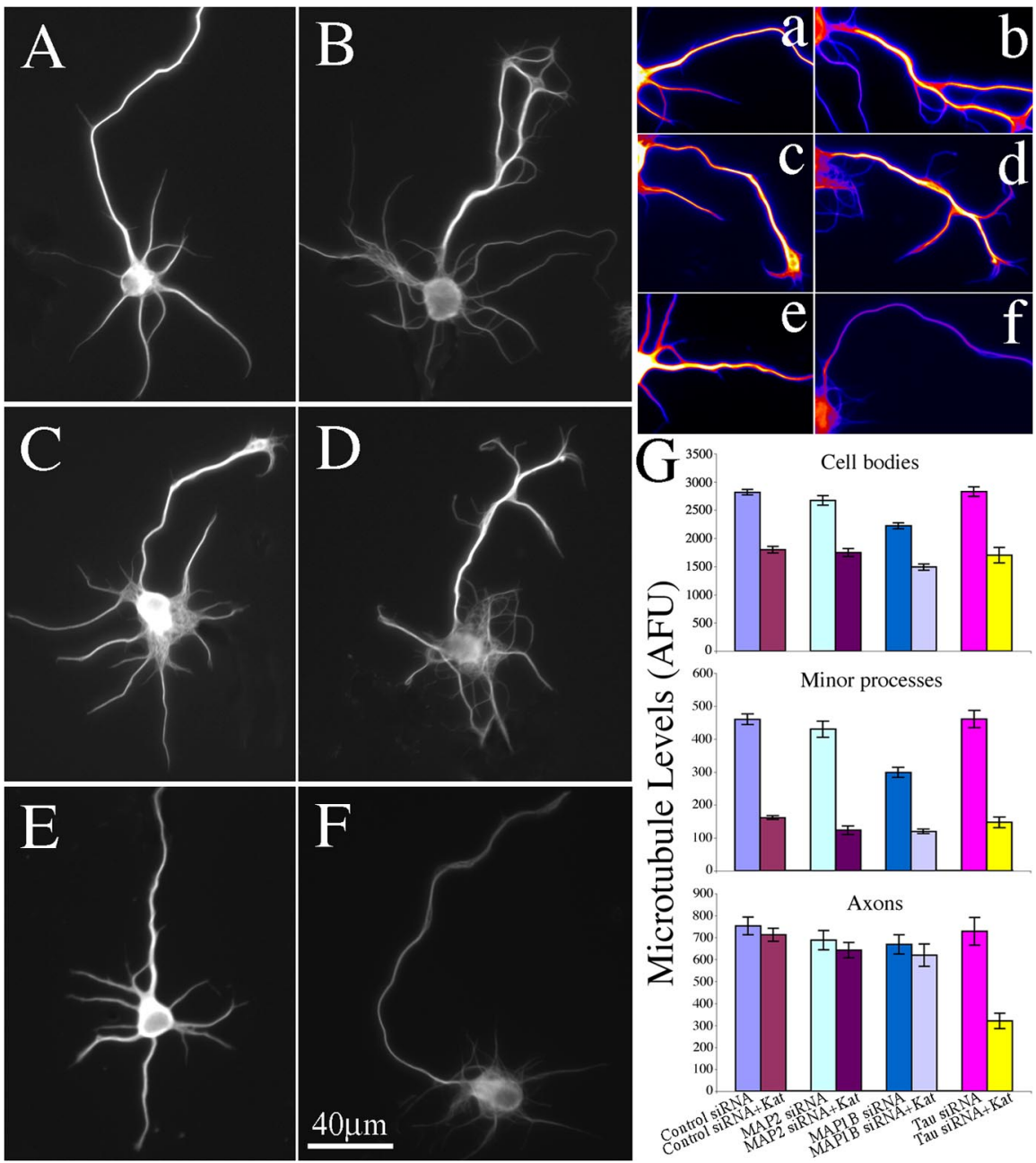

Figure 7. Depletion of tau from cultured hippocampal neurons increases sensitivity to katanin-induced microtubule severing whereas depletion of MAP1b or MAP2 does not. $\boldsymbol{A}-\boldsymbol{F}$, Microtubule immunostains of stage III hippocampal neurons. $\boldsymbol{A}, \mathrm{A}$ neuron transfected with control siRNA and GFP. B, A neuron transfected with control siRNA and P60-katanin. $C$, A neuron transfected with MAP1b siRNA and GFP. D, A neuron transfected with MAP1b siRNA and P60-katanin. $\boldsymbol{E}$, A neuron transfected with tau siRNA and GFP (axon is directed upwards in the panel). $\boldsymbol{F}$, A neuron transfected with tau siRNA and P60-katanin. Note that the axons show no diminution in fluorescence intensity as a result of overexpression of P60-katanin in either control siRNA or MAP1b siRNA-treated neurons. However, a dramatic diminution was detected in tau-depleted neurons overexpressing P60-katanin. $\boldsymbol{a}-\boldsymbol{f}$, Glow-scale pseudocolored images of the axons in $\boldsymbol{A}-\boldsymbol{F}$, with white indicating the highest level, purple indicating the lowest level, and shades of red, orange, and yellow indicating intermediate levels. $\boldsymbol{G}$. The quantification of microtubule mass in cell bodies, in minor processes, and in axons of stage III neurons in each group. 0verexpression of P60-katanin in either control group or the three MAP siRNA-treated groups leads to a 33-40\% diminution in microtubule mass from cell bodies $(p<0.01)$. Overexpression of P60-katanin in either control group or the three MAP siRNA-treated groups leads to a $60-71 \%$ diminution in microtubule mass from minor processes $(p<0.0001)$. The microtubules in axons of tau depleted neurons were very sensitive to overexpression of P60-katanin, leading to a $63 \%$ diminution $(p<0.001)$. The microtubules in axons of the other three groups showed no diminution as a result of the overexpression of P60-katanin $(p>0.05)$. Error bars represent SE. AFU, Arbitrary fluorescence units; Kat, katanin.

were stabilized), providing support for the conclusion that MAP1b is not a likely candidate for protecting axonal microtubules from severing.

These observations suggest that the protection phenomenon may be specific to the tau/MAP2/MAP4 family of related MAPs. Neurons express a variety of tau and MAP2 isoforms (Dehmelt and Halpain, 2004; Luo et al., 2004), and it has been known for many years that neurons compartmentalize many of these isoforms (Peng et al., 1986; Ferreira et al., 1987). Recent work suggests that overexpression of these MAPs can attenuate the interaction of molecular motor proteins with the microtubule lattice (Mandelkow et al., 2004), and the results presented here suggest a similar scenario for the microtubule severing proteins. The fact that axonal microtubules are so much more resistant to katanin than microtubules in any other compartment of the neuron may relate to the levels of the particular MAP in the axon versus other neuronal compartments, the phosphorylation state of the MAP in each neuronal compartment, or to some aspect of the protection not revealed by the RFL6 studies. In addition, we should note that neurons express a variety of other proteins that can bind microtubules, such as doublecortin (Francis et al., 1999) and stable-tubule-only-polypeptide (Slaughter and Black, 2003), and we have not exhaustively determined whether these various proteins might be able to afford protection against katanin. Instead, we decided to shift our attention to bona fide neurons to directly test our hypothesis that tau is the chief protector of axonal microtubules against severing.

For these studies on primary neurons, we used a contemporary siRNA approach to deplete tau, MAP2, or MAP1b from cultured hippocampal neurons, after which we replated the neurons to allow them to extend processes after depleting the MAP. MAP protein was depleted by $95-99 \%$ relative to controls. P60-katanin was then overexpressed for $12 \mathrm{~h}$ to evaluate for potential changes in the sensitivity of the microtubules to severing. We were initially surprised that the neurons, depleted of any one of these MAPs, were fully capable of extending lamellipodia, minor processes, and an axon. The time table of differentiation was noticeably slower after depletion of MAP1b, with fewer cells differentiating axons by the third day after replating. The time table of differentiation was unaffected by depletion of tau or MAP2. The length of the axon on day 3 was $\sim 10 \%$ shorter after depletion of tau or MAP2 but $\sim 21 \%$ shorter after depletion of MAP1b. The only other differences we detected, compared with controls, was that the microtubule levels were diminished by $20 \%$ in the cell bodies (but not axons or minor processes) of neurons depleted of MAP1b, and the microtubules were more unevenly distributed in their axons, with a higher density more distally. These results indicate that cultured hippocampal neurons are able to differentiate fairly normally in the absence of any one of these MAPs, at least to the point of axonal differentiation.

As reported previously (Yu et al., 2005), overexpression of P60-katanin for $12 \mathrm{~h}$ results in dramatic loss of microtubule mass from the cell body and minor processes of control neurons, but not from the axon. After depletion of MAP1b or MAP2, this same result was obtained. However, after depletion of tau, the loss of microtubule mass from the axon was equally dramatic as anywhere else in the neuron, indicating that tau is indeed the factor 
in the axon that is responsible for rendering the microtubules more resistant to katanin. We do not think that tau is simply stabilizing the microtubules against disassembly after severing, because depletion of tau does not, within itself, diminish the microtubule mass, nor does it render axonal microtubules any more sensitive to depolymerization by drugs such as nocodazole (Tint et al., 1998).

The fact that $12 \mathrm{~h}$ of P60-katanin overexpression after tau depletion does not entirely eliminate all microtubules from the cell body or minor processes (or dendrites) (Yu et al., 2005) suggests that tau is not the only factor that protects the microtubules. MAP2, the isoforms of which are widespread in developing neurons, although not the chief protector, could presumably afford some protection, and as noted above, there may be other microtubule-binding proteins with the capacity to protect against severing that we have not studied. The idea that tau is not the only protector in the axon is supported by the fact that there were no obvious changes in the axonal microtubules, at least at the immunofluorescence level, when tau was depleted without an experimental increase in P60-katanin levels above the endogenous levels. Either the small amount of tau that is not completely depleted during siRNA treatment is sufficient to provide some degree of protection in our experimental regimen, or other molecules contribute to the protection.

The axon is endowed with very long microtubules, which are presumably important for the axon to obtain such great lengths. These long microtubules act as highways for vesicle transport and also serve to offset potential retraction of the axon (Baas and Ahmad, 2001). Our studies suggest that tau is important for ensuring that katanin does not have unfettered access to the microtubules and is thereby critical for the maintenance of the axonal microtubule array. We suspect that this same mechanism applies to spastin, another microtubule-severing protein present in the neuron (Errico et al., 2002; Evans et al., 2005; Roll-Mecak and Vale, 2005). These ideas are particularly exciting in light of the category of human diseases known as tauopathies, which involve the gradual dissociation of tau from the microtubules (Stoothoff and Johnson, 2005). We propose that the sustained loss of tau from axonal microtubules over time renders them more sensitive to endogenous severing proteins, thus causing the microtubule array to gradually disintegrate in tauopathies such as Alzheimer's disease.

\section{References}

Ahmad FJ, Yu W, McNally FJ, Baas PW (1999) An essential role for katanin in severing microtubules in the neuron. J Cell Biol 14:305-315.

Baas PW, Ahmad FJ (2001) Force generation by cytoskeletal motor proteins as a regulator of axonal elongation and retraction. Trends Cell Biol 11:244-249.

Baas PW, Qiang L (2005) Neuronal microtubules: when the MAP is the roadblock. Trends Cell Biol 15:183-187.

Brugg B, Reddy D, Matus A (1993) Attenuation of microtubule-associated protein 1B expression by antisense oligodeoxynucleotides inhibits initiation of neurite outgrowth. Neuroscience 52:489-496.

Caceres A, Kosik KS (1990) Inhibition of neurite polarity by tau antisense oligonucleotides in primary cerebellar neurons. Nature 343:461-463.

Caceres A, Potrebic S, Kosik KS (1991) The effect of tau antisense oligonucleotides on neurite formation of cultured cerebellar macroneurons. J Neurosci 11:1515-1523.

Caceres A, Mautino J, Kosik KS (1992) Supression of MAP2 in cultured cerebellar macroneurons inhibits minor neurite formation. Neuron 9:607-618.

Cairns NJ, Lee VM, Trojanowski JQ (2005) The cytoskeleton in neurodegenerative diseases. J Pathol 204:438-449.

Chang W, Gruber D, Chari S, Kitazawa H, Hamazumi Y, Hisanaga S, Bulinski
JC (2001) Phosphorylation of MAP4 affects microtubule properties and cell cycle progression. J Cell Sci 114:2879-2887.

Chapin SJ, Bulinski JC (1991) Non-neuronal 210 x 10(3) Mr microtubule associated-protein (MAP4) contains a domain homologous to the microtubule-binding domains of neuronal MAP2 and tau. J Cell Sci 98:27-36.

Chapin SJ, Lue CM, Yu MT, Bulinski JC (1995) Differential expression of alternatively spliced forms of MAP4: a repertoire of structurally different microtubule-binding domains. Biochemistry 34:2289-2301.

Dawson HN, Ferreira A, Eyster MV, Ghoshal N, Binder LI, Vitek MP (2001) Inhibition of neuronal maturation in primary hippocampal neurons from tau deficient mice. J Cell Sci 114:179-1187.

Dehmelt L, Halpain S (2004) Actin and microtubules in neurite initiation: are MAPs the missing link? J Neurobiol 58:18-33.

Dent EW, Callaway JL, Szebenyi G, Baas PW, Kalil K (1999) Reorganization and movement of microtubules in growth cones and developing interstitial branches. J Neurosci 9:8894-8904.

DiTella MC, Feiguin F, Carri N, Kosik KS, Caceres A (1996) MAP1B/TAU functional redundancy during laminin-enhanced axonal growth. J Cell Sci 109:467-477.

Errico A, Ballabio A, Rugarli EI (2002) Spastin, the protein mutated in autosomal dominant hereditary spastic paraplegia, is involved in microtubule dynamics. Hum Mol Genet 11:153-163.

Evans KJ, Gomes ER, Reisenweber SM, Gundersen GG, Lauring BP (2005) Linking axonal degeneration to microtubule remodeling by Spastinmediated microtubule severing. J Cell Biol 14:599-606.

Ferreira A, Busciglio J, Caceres A (1987) An immunocytochemical analysis of the ontogeny of the microtubule-associated proteins MAP- 2 and Tau in the nervous system of the rat. Brain Res 431:9-31.

Francis F, Koulakoff A, Boucher D, Chafey P, Schaar B, Vinet MC, Friocourt G, McDonnell N, Reiner O, Kahn A, McConnell SK, Berwald-Netter Y, Denoulet P, Chelly J (1999) Doublecortin is a developmentally regulated, microtubule-associated protein expressed in migrating and differentiating neurons. Neuron 23:247-256.

Gamblin TC, Nachmanoff K, Halpain S, Williams Jr RC (1996) Recombinant microtubule-associated protein $2 \mathrm{c}$ reduces the dynamic instability of individual microtubules. Biochemistry 35:12576-12586.

Gonzalez-Billault C, Engelke M, Jimenez-Mateos EM, Wandosell F, Caceres A, Avila J (2002) Participation of structural microtubule-associated proteins (MAPs) in the development of neuronal polarity. J Neurosci Res 67:713-719.

Goold RG, Gordon-Weeks PR (2005) The MAP kinase pathway is upstream of the activation of GSK3beta that enables it to phosphorylate MAP1B and contributes to the stimulation of axon growth. Mol Cell Neurosci 28:524-534.

Gordon-Weeks PR, Fischer I (2000) MAP1B expression and microtubule stability in growing and regenerating axons. Microsc Res Tech 48:63-74.

Hall GF, Yao J, Lee G (1997) Human tau becomes phosphorylated and forms filamentous deposits when overexpressed in lamprey central neurons in situ. Proc Natl Acad Sci USA 94:4733-4738.

Harada A, Teng J, Takei Y, Oguchi K, Hirokawa N (2002) MAP2 is required for dendrite elongation, PKA anchoring, and proper PKA signal transduction. J Cell Biol 158:541-549.

He Y, Francis F, Myers KA, Yu W, Black MM, Baas PW (2005) Role of cytoplasmic dynein in the axonal transport of microtubules and neurofilaments. J Cell Biol 168:697-703.

Johnson GV, Stoothoff WH (2004) Tau phosphorylation in neuronal cell function and dysfunction. J Cell Sci 117:5721-5729.

Karabay A, Yu W, Solowska JM, Baird DH, Baas PW (2004) Axonal growth is sensitive to the levels of katanin, a protein that severs microtubules. J Neurosci 24:5778-5788.

Kawauchi T, Chihama K, Nishimura YV, Nabeshima Y, Hoshino M (2005) MAP1B phosphorylation is differentially regulated by Cdk5/p35, Cdk5/ p25, and JNK. Biochem Biophys Res Commun 331:50-55.

Krichevsky A, Kosik K (2002) RNAi functions in cultured mammalian neurons. Proc Natl Acad Sci USA 99:11926-11929.

Lee G, Rook SL (1992) Expression of tau protein in non-neuronal cells: microtubule binding and stabilization. J Cell Sci 102:227-237.

Leger JG, Brandt R, Lee G (1994) Identification of tau protein regions required for process formation in PC12 cells. J Cell Sci 107:3403-3412.

Lu M, Kosik KS (2001) Competition for microtubule-binding with dual expression of tau missense and splice isoforms. Mol Biol Cell 12:171-184. 
Luo MH, Tse SW, Memmott J, Andreadis A (2004) Novel isoforms of tau that lack the microtubule-binding domain. J Neurochem 90:340-351.

Mandelkow EM, Thies E, Trinczek B, Biernat J, Mandelkow E (2004) MAPK/PAR kinase is a regulator of microtubule-dependent transport in axons. J Cell Biol 167:99-110.

Mandell JW, Banker GA (1996) A spatial gradient of tau protein phosphorylation in nascent axons. J Neurosci 16:5727-5740.

McNally FJ, Vale RD (1993) Identification of katanin, an ATPase that severs and disassembles stable microtubules. Cell 75:419-429.

McNally KP, Bazirgan OA, McNally FJ (2000) Two domains of p80 katanin regulate microtubule severing and spindle pole targeting by p 60 katanin. J Cell Sci 113:1623-1633.

McNally KP, Buster D, McNally FJ (2002) Katanin-mediated microtubule severing can be regulated by multiple mechanisms. Cell Motil Cytoskeleton $53: 337-349$.

Meichsner M, Doll T, Reddy D, Weisshaar B, Matus A (1993) The low molecular weight form of microtubule-associated protein 2 is transported into both axons and dendrites. Neuroscience 54:8738-8780.

Peng I, Binder LI, Black MM (1986) Biochemical and immunological analyses of cytoskeletal domains of neurons. J Cell Biol 102:252-262.

Roll-Mecak A, Vale R (2005) The Drosophila homologue of the hereditary spastic paraplegia protein, spastin, severs and disassembles microtubules. Curr Biol 15:650-655.

Rossi J, Eckstein F (2003) RNAi impacts the world of antisense. Oligonucleotides 13:279-280.

Sambrook J, Fritsch EF, Maniatis T (1989) Molecular cloning. A Laboratory Manual, Ed 2 (Janssen K, ed), pp 18.47-18.71. Cold Spring Harbor, NY: Cold Spring Harbor Laboratory.
Seitz A, Kojima H, Oiwa K, Mandelkow EM, Song YH, Mandelow E (2002) Single-molecule investigation of the interference between kinesin, tau, and MAP2c. EMBO J 21:4896-4905.

Slaughter T, Black MM (2003) STOP (stable-tubule-only-polypeptide) is preferentially associated with the stable domain of axonal microtubules. J Neurocytol 32:399-413.

Stoothoff WH, Johnson GV (2005) Tau phosphorylation: physiological and pathological consequences. Biochim Biophys Acta 1739:280-297.

Takemura R, Okabe S, Umeyama T, Kanai Y, Cowan NJ, Hirokawa N (1992) Increased microtubule stability and alpha tubulin acetylation in cells transfected with microtubule-associated proteins MAP1B, MAP2 or tau. J Cell Sci 103:953-964.

Teng J, Takei J, Nakata T, Chen J, Hirokawa N (2001) Synergistic effects of MAP2 and MAP1b knockout in neuronal migration, dendritic outgrowth, and microtubule organization. J Cell Biol 155:65-76.

Tint I, Slaughter T, Fischer I, Black MM (1998) Acute inactivation of tau has no effect on dynamics of microtubules in growing axons of cultured sympathetic neurons. J Neurosci 18:8660-8673.

Vale RD (1991) Severing of stable microtubules by a mitotically activated protein in Xenopus egg extracts. Cell 64:827-839.

Wang L, Brown A (2002) Rapid movement of microtubules in axons. Curr Biol 12:1496-1501.

Yu W, Baas PW (1994) Changes in microtubule number and length during axon differentiation. J Neurosci 14:2818-2829.

Yu W, Solowska JM, Qiang L, Karabay A, Baird D, Baas PW (2005) Regulation of microtubule severing by katanin subunits during neuronal development. J Neurosci 25:5573-5583. 